Original Research Paper

\title{
Tissue Engineering of Articular Cartilage: A Mini-Review
}

\author{
${ }^{1}$ Radoslav Zamborsky, ${ }^{2}$ Jana Rusnakova and ${ }^{3}$ Andreas Nicodemou \\ ${ }^{1}$ Department of Traumatology, Faculty of Medicine, Slovak Medical University, Bratislava, Slovakia \\ ${ }^{2}$ Institute of Histology and Embryology, \\ ${ }^{3}$ Institute of Medical Biology, Genetics and Clinical Genetics, \\ Faculty of Medicine, Comenius University, Bratislava, Slovakia
}

\author{
Article history \\ Received: 23-07-2014 \\ Revised: 01-10-2014 \\ Accepted: 10-10-2014 \\ Corresponding Author: \\ Radoslav Zamborsky, \\ Department of Traumatology, \\ Faculty of Medicine, Slovak \\ Medical University, Bratislava, \\ Slovakia \\ Email: radozamborsky@gmail.com
}

\begin{abstract}
Damage of articular cartilage due to congenital anomaly, injury or pathological process may lead in decreasing of life quality of affected patients. In many cases, conventional therapeutical approaches may not bring expected results. Tissue engineering by the combination of material technology and cell-based therapy may represent hope for these patients. The main goal of this review article is to summarize current knowledge about biological characteristics of somatic stem cells, chondro-inductive substances and materials in respect to regeneration of articular cartilage.
\end{abstract}

Keywords: Articular Cartilage, Somatic Stem Cells, Growth Factors, Biomateriasls, Regenerative Medicine

\section{Introduction}

Recently, despite the advances in medicine, damaged articular cartilage as a consequence of inborn defects, injury or pathological process still represent serious medical problem. It may lead in gradual imobilization and decreasing of life quality in affected individuals. Mentioned is mainly contributed to low self-healing potential of mature articular cartilage due to intrinsic properties. Articular cartilage is aneural and avascular tissue with low density of cells. Moreover, high level of protease inhibitors inhibit tissue repair (Zhang et al., 2009a).

Small defects are usually regenerated by the migration of chondrocytes to cartilage lesions. They synthesize new ECM components of cartilage. In case of large scale and deep defects, this process is complicated by low cell density, by low mitotic potential of chondrocytes as well as by high level of protease inhibitors. It results in formation of the biomechanically insufficient fibrillar cartilage (Mobasheri et al., 2009). Unfortunately, current treatment techniques for cartilage reparation are insufficient and it is not possible to obtain expected results.

The tissue engineering offers new concept to solve this serious problem. The first cell-based therapy for articular cartilage treatment was introduced by Brittberg et al. (1994) who injected autologous chondrocytes into the lesion covered by periosteal flap in 23 people with deep cartilage defects in the knee.
This lead into formation of the hyaline-like cartilage. However, this approach showed some disadvantages, including reacquisition of chondrocyte phenotype during in vitro expansion and non-uniform distribution of cells due to gravitational force.

More recently, it was shown that somatic stem cell undergo the process of chondrogenic differentiation under propper conditions, both in vitro and in vivo. They should be obtained from different tissue sources and easily expended in vitro (Danisovic et al., 2011). Therefore, the great hope is addressed on their utilization in relation to repairing damaged articular cartilage.

The main goal of the present article is to review the current status and advances of the cartilage tissue engineering with respect to their potential application in orthopaedic surgery and traumatology.

\section{Histology of Articular Cartilage}

Articular cartilage is a type of hyaline cartilage (Fig. 1) and belongs to connective tissues. It is composed of abundant Extracellular Matrix (ECM) which contains predominantly collagen type II, chondroitine sulphate and proteoglycans. Articular cartilage is characterized by zonality. Different organization of collagen fibbers and cross-linking to other components influence its biomechanical properties (Sophia Fox et al., 2009).

The ECM is very poor for cells-chondrocytes. On the periphery they have elliptic morphology. Chondrocytes 
localized inside ECM are of round morphology with average size of 10-30 $\mu \mathrm{m}$. They formed isogenous cell groups in cavities, called cartilage lacunae (Mescher, 2013). They are responsible for production of ECM components. Moreover, they are involved in the maintaining and remodelling of the articular cartilage (Cucchiarini et al., 2012; Danisovic et al., 2013).

\section{Cell Sources for Articular Cartilage Tissue Engineering}

Chondrocytes are the cells of first choice for cartilage tissue engineering, because they occurs within articular cartilage in vivo. They have been used in all current Autologous Chondrocyte Implantation (ACI) procedures. Chondrocytes have been isolated from different sources, including low load-bearing area of knee cartilage and auricular cartilage (Beris et al., 2012; Malicev et al., 2009). However, this technique has some limits due to low mitotic potential and senescence of chondrocytes. Moreover, they undergo dedifferentiation process when cultured in vitro, gradually changing their morphology to a fibroblast-like shape and the production of type II collagen is replaced by the production of collagen type I. This problem may be overlapped by adding specific growth factors.

Somatic Stem Cells (SSCs) because of their biological characteristics represent another promising tool for cartilage tissue engineering. SSCs are undifferentiated cells with unique potential of selfrenewing and plasticity (Danisovic et al., 2011).

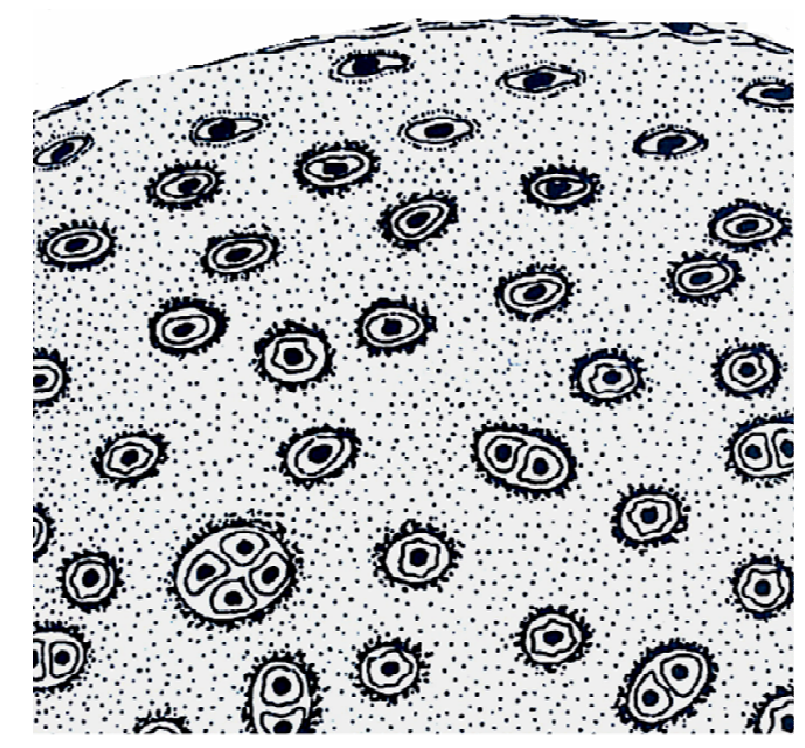

Fig. 1. Schematic drawing of articular (hyaline) cartilage containing abundant ECM and chondrocytes
They have been isolated and expanded in vitro from different tissue sources, including bone marrow, adipose tissue, muscles, dental pulp, umbilical cord Wharton's jelly (Odabas et al., 2014; Danisovic et al., 2011; Varga et al., 2011; Zhang et al., 2009b).

SSCs are adherent cells, which express specific surface antigens, including Stro-1, CD29, CD44, CD73, CD90 and CD105. They are negative for CD31, CD34 and CD45. Moreover, Kestendjieva et al. (2008) demonstrated expression of antiapoptotic proteinsurvivin, which is also expressed in most human cancers (Adamkov et al., 2012; 2011).

Since the end of 1990s, a lot of studies focused on chondrogenic differentiation were done. It was demonstrated that SSCs derived from bone marrow cultured in high densities with culture medium containing TGF- $\beta 1$ lead into increased expression of collagen type II and $\mathrm{X}$ typical for articular cartilage in vivo. On other hand the expression of collagen type I was significantly decreased (Fortier et al., 2011). More recently, the spontaneous chondrogenesis of SSCs was also proved in case of pellet cultures (Havlas et al., 2011).

Results from clinical case reports and clinical trials indicated that SSCs may positively affect the cartilage repair. Kuroda et al. (2007) isolated autologous SSCs from bone marrow. After their in vitro cultivation, SSCs were embedded within a collagen gel, which was applied to cartilage defect and covered by periosteal flap. After 7 months the deffect was filled with a hyaline cartilage. Centeno et al. (2008) injected suspension of autologous SSCs isolated from bone marrow into the subject's knee with MRI proven degenerative joint disease. After 24 weeks it resulted in significant cartilage growth, decreased pain and increased joint mobility of patient.

More recently, the extensive study was conducted to compare the clinical effect of autologous chondrocytes implantation to patients treated with autologous SSCs from bone marrow. About 72 patients were divided in two groups, 36 were treated by chondrocytes and 36 with SSCs. The results proved that both types of cells had similar effect on cartilage defect treatment, but in case of SSCs it required 1 less knee surgery, reduced costs and minimized donor-site morbidity (Nejadnik et al., 2010).

Induced Pluripotent Stem Cells (IPSCs) significantly expand options of cell therapy. IPSCs are type of pluripotent cells that can be generated directly from terminally differentiated somatic cells (Csobonyeiova et al., 2013).

The first evdence that differentiated somatic cells may be reprogrammed into undifferentiated cells have been demonstrated by somatic nuclear transfer (Wilmut et al., 1997). The first IPSCs have been prepared from fibroblasts of mouse with using 4 transcription factors Oct4, Sox2, c-myc a Klf4 (Takahashi and Yamanaka, 
2013). This combination did not work in human. Moreover, utilization of c-myc led to malignant transformation (Shimizu et al., 2010). The first human IPSCs have been prepared by two independent research groups in USA (University of Wisconsin-Madison) and Japan (Kyoto University) from fibroblasts by using of Oct4, Sox2, Nanog a LIN28 (Yu et al., 2007; Takahashi et al., 2007). IPSCs displayed similar morphological and biological properties as embryonic stem cells, including expression of specific antigens, increased proliferation and telomerase activity and pluripotency. Moreover, they were able to produce embryoid bodies and teratomas (Yu et al., 2007). Since these discoveries, IPSCs belong to most promising tools of regenerative and personalized medicine. For overview of their biological properties, possibilities of preparation and utilization in biomedicine check article by (Csobonyeiova et al., 2013).

IPSCs were also studied in context of cartilage tissue engineering. Diekman et al. (2012) fabricated artificial cartilage tissue from IPSCs using micromass culture for purification of chondrogenic cells and pellet culture system with TGF- $\beta 3$ to induce chondrogenic differentiation in vitro. Their results proved increased expression of collagen type II and aggrecan. More recently, Ko et al. (2014) demonstrated successful chondrogenesis and regeneration of damaged cartilage with human iPSCs. Chondrogenic differentiation was induced by using alginate hydrogel culture system. Afterwards, micro aggregates of alginate constructs were implanted in osteochondral defects created on the patellar groove of immunosuppressed rats. After 21 days, they observed greater glycosaminoglycan contents and better chondrocytic features including lacuna and abundant matrix formation. However, further studies are necessary for translation of IPSCs into clinical practice, mainly focused on their biological safety.

\section{Biomaterials for Cartilage Tissue Engineering}

Cartilage tissue engineering employs many biomaterials of natural or synthetic origin (Table 1). They may be in form of hydrogel, sponges, fibrous meshes and nanofibres. The crucial characteristics are their non-toxicity and biocompatibility. Other characteristics, such as porosity (size and orientation of pores) and structural strenght also influnce their final utilization (Liu et al., 2013).

The most commonly used natural material is collagen which belongs to basic constituents of cartilage in vivo. The mechanical properties of collagen-based scaffolds may be easily controlled by chemical modifications (Danisovic et al., 2013). It was shown that chondrocytes cultured within collagen scaffolds maintain their original phenotype and production of Glycosaminoglycans (GAGs) under in vitro conditions. Moreover, several authors provide evidence of strong chondroinductive effect on SSCs (Zhang et al., 2012; Zheng et al., 2010).
Table 1. Types of biomaterials used in cartilage tissue engineering

\begin{tabular}{ll}
\hline Natural polymers & Synthetic polymers \\
\hline Agarose & Poly ( $\alpha$-hydroxy esters) \\
Alginate & Poly (ethylene glycol/oxide) \\
Cellulose & Poly (NiPAAm) \\
Collagen & Poly (propylene fumarate) \\
Chitosan & Poly (urethane) \\
Fibrin & Poly (vinyl alcohol) \\
Gelatine & \\
Hyaluronic acid & \\
Silk fibroin & \\
\hline
\end{tabular}

Hyaluronic Acid (HA) is another natural biopolymer studied in the context of cartilage tissue engineering. Ha also occurs in native cartilage and should be used itself or in combination with other biomaterials. It was shown that chondrocytes cultured within HA hydrogels are forced to produce collagen type II and aggrecan typical for hyaline cartilage. Furthermore, SSCs cultured within photo-cross-linked HA hydrogel undergo chondrogenic differentiation (Chung and Burdick, 2009).

Other natural biomaterials that have been used in cartilage tissue engineering include alginate, agarose, chitosan, silk fibroin (Nooeaid et al., 2012; Yu et al., 2012).

Besides the above mentioned natural biopolymers, a variety of synthetic polymers may be applied in cartilage tissue engineering. When compared with natural biomaterials, they have several advantages, including highly controlled physical characteristics, consistency, uniformity and unlimited production (Yu et al., 2012).

The most widely used are Polylactic Acid (PLA) and Polyglycolic Acid (PGA) (and their co-polymer). Both of them belong to biodegradable polymers. It was demonstrated that they increase chondrocyte proliferation and GAGs production. Moreover, several authors provide evidence of their effect on SSCs proliferation and chondrogenic differentiation (Foldberg et al., 2012; Xue et al., 2012).

Poly (Ethylene Glycol) (PEG) and its derivates, mainly in form of hydrogel wer also evaluated in respect to cartilage regeneration. Hwang et al. (2010) demonstrated that chondrocytes cultured within PEG scaffold remain alive and underwent chondrogenic differentiation. More recently, Cui et al. (2014) prepared cartilage constructs by using 3D printing technology. They used PEG-based scaffold with chondrocytes and demonstrated their full viability and prominent production of collagen type II and GAGs.

There are a lot of other synthetic materials that have been studied in respect to cartilage tissue engineering, e.g., poly ( $\alpha$-hydroxy esters), poly (propylene fumarate), poly (urethane) (Yu et al., 2012).

\section{Growth Factors}

Growth factors play pivotal role in the process of chondogenesis. They represent a group of biologically 
active polypeptides that may affect cell proliferation and differentiation. In the hyaline cartilage, specific growth factors regulate homeostasis, integrity and development. The effect of growth factor on chondrogenic differentiation may differ depending on its dose, specific cell type and cell differentiation (Yu et al., 2012).

Most studied growth factors in respect to cartilage tissue engineering include members of Transforming Growth Factor- $\beta$ (TGF- $\beta$ ) superfamily, Fibroblast Growth Factor (FGF) family and Insulin-like Growth Factor 1 (IGF-1).

TGF- $\beta$ superfamily contains at least 20 members in vertebrates. The best candidates for cartilage tissue engineering are TGF- $\beta 1$, TGF- $\beta 3$, BMP-2, BMP-4, BMP-7 and CDMP-1 (also known as GDF-5). TGF- $\beta 1$ promotes the synthetic activity of chondrocytes and decreases catabolic activity of IL-1 and MMPs in vivo. TGF- $\beta 3$ enhances synthesis of sulphated GAGs. BMP2 stimulates synthesis of cartilage-specific ECM. BMP4 is essential for normal embryogenic development and exhibits osteogenic and chondrogenic potential in vivo. BMP-7 has significant anabolic activity by which protects cartilage against damage. GDF-5 increases proliferation of chondrocytes as well as play important roles during the development of skeleton and joints (Fortier et al., 2011).

FGF family contains at least 23 members in vertebrates. Mainly FGF-2, FGF-4, FGF-8 and FGF-18 were studied in the context of cartilage tissue engineering. It was shown, that FGF-2 promotes the proliferation of chondrocytes in vivo. FGF-2 with FGF-4 and FGF-8 are involved in the process of anabolic pathways activation which leads to decrease of aggrecanase effect after cartilage load. Furthermore, FGF-18 is involved in a variety of biological processes, including embryonic development, cell growth, morphogenesis and tissue repair (Ellman et al., 2013).

IGF-1 stimulates chondrocytes to synthesize cartilage-specific ECM and decreases catabolic responses. Moreover, it was demonstrated that IGF-1 has an additive effect on increase of cartilage matrix synthesis when acts with TGF- $\beta 1$, BMP2 and BMP7 (An et al., 2010; Gow et al., 2010).

\section{Conclusion}

Recently, cartilage tissue engineering provides new promising approach which should be used in healing patients with damaged articular cartilage. It combines different types of cells (chondrocytes and stem cells), various scaffolding materials and appropriate growth factors to prepare fully biologically active artificial cartilage tissue. However, prior to translation into clinical practice the further studies have to be carried out, mainly focused on safety of stem cells expanded under in vitro conditions. Considerable progress can be expected also in field of material technology, mainly in combination with 3D bioprinting.

\section{Acknowledgment}

The present study was partly supported by the grant of Ministry of Health of the Slovak Republic No. 2012/4-UKBA-4.

\section{Author's Contributions}

All authors equally contributed in this work.

\section{Ethics}

This article is original and contains unpublished material. The corresponding author confirms that all of the other authors have read and approved the manuscript and no ethical issues involved.

\section{References}

Adamkov, M., E. Halasova, J. Rajcani, M. Bencat and D. Vybohova et al., 2011. Relation between expression pattern of p53 and survivin in cutaneous basal cell carcinomas. Med. Sci. Monit., 17: BR74-80. DOI: $10.12659 /$ MSM.881442

Adamkov, M., K. Kajo, D. Vybohova, J. Krajcovic and F. Stuller et al., 2012. Correlations of survivin expression with clinicomorphological parameters and hormonal receptor status in breast ductal carcinoma. Neoplasma, 59: 30-37. DOI: 10.4149/neo_2012_004

An, C., Y. Cheng, Q. Yuan and J. Li, 2010. IGF-1 and BMP-2 induces differentiation of adipose-derived mesenchymal stem cells into chondrocytes-like cells. Ann. Biomed Eng., 38: 1647-1654.

DOI: 10.1007/s10439-009-9892-X

Beris, A.E., M.G. Lykissas, I. Kostas-Agnantis and G.N. Manoudis, 2012. Treatment of full-thickness chondral defects of the knee with autologous chondrocyte implantation: A functional evaluation with long-term follow-up. Am. J. Sports Med., 40: 562-567. DOI: 10.1177/0363546511428778

Brittberg, M., A. Lindahl, A. Nilsson, C. Ohlsson and O. Isaksson et al., 1994. Treatment of deep cartilage defects in the knee with autologous chondrocyte transplantation. N Engl. J. Med., 331: 889-895. DOI: 10.1056/NEJM199410063311401

Centeno, C.J., D. Busse, J. Kisiday, C. Keohan and M. Freeman at al., 2008. Increased knee cartilage volume in degenerative joint disease using percutaneously implanted, autologous mesenchymal stem cells. Pain Physician, 11: 343-53.

PMID: 18523506 
Chung, C. and J.A. Burdick, 2009. Influence of threedimensional hyaluronic acid microenvironments on mesenchymal stem cell chondrogenesis. Tissue Eng. Part A., 15: 243-254. DOI: 10.1089/ten.tea.2008.0067

Csobonyeiova, M., S. Polak and L. Danisovic, 2013. Induced pluripotent stem cells and their implication for biomedical sciences. OnLine J. Biol. Sci.., 13: 106-114. DOI: 10.3844/ojbsci.2013.106.114

Cucchiarini, M., J.K. Venkatesan, M. Ekici, G. Schmitt and H. Madry, 2012. Human mesenchymal stem cells overexpressing therapeutic genes: From basic science to clinical applications for articular cartilage repair. Biomed Mater Eng., 22: 197-208. DOI: 10.3233/BME-2012-0709

Cui, X., G. Gao, T. Yonezawa and G. Dai, 2014. Human cartilage tissue fabrication using three-dimensional inkjet printing technology. J. Vis. Exp. PMID: 24961492

Danisovic, L., I. Varga, S. Polák, B. Bajciková and M. Adamkov et al., 2011. Biological and morphological characterization of in vitro expanded human musclederived stem cells. Tsitologiia, 53: 482-487. PMID: 21870504

Danisovic, L., Z. Novakova-Varchulova, M. Bohac, D. Bakos and J. Vojtassak, 2013. In vitro testing of modified collagen/hyaluronan/beta-glucan scaffold. OnLine J. Biol. Sci.., 13: 40-45.

DOI: $10.3844 /$ ojbsci.2013.35.40

Diekman, B.O., N. Christoforou, V.P. Willard, H. Sun and J. Sanchez-Adams et al., 2012. Cartilage tissue engineerin using differentiated and purified induced pluripotent stem cells. PNAS, 109: 19172-19177. DOI: $10.1073 /$ pnas. 1210422109

Ellman, M.B., D. Yan, K. Ahmadinia, D. Chen and H.S. An et al., 2013. Fibroblast growth factor control of cartilage homeostasis. J. Cell Biochem., 114: 735-742. DOI: 10.1002/jcb. 24418

Foldberg, S., M. Petersen, P. Fojan, L. Gurevich and T. Fink et al., 2012. Patterned poly (lactic acid) films support growth and spontaneous multilineage gene expression of adipose-derived stem cells. Colloids Surf B Biointerfaces, 93: 92-99. DOI: 10.1016/j.colsurfb.2011.12.018

Fortier, L.A., J.U. Barker, E.J. Strauss, T.M. McCarrel and B.J. Cole, 2011. The role of growth factors in cartilage repair. Clin. Orthop Relat Res., 469: 27062715. DOI: $10.1007 /$ s 1 1999-011-1857-3

Gow, D.J., D.P. Sester and D.A. Hume, 2010. CSF-1, IGF-1 and the control of postnatal growth and development. J. Leukoc Biol., 88: 475-481. DOI: $10.1189 / \mathrm{jlb} .0310158$

Havlas, V., P. Kos, P. Jendelova, P. Lesny and T. Tre et al., 2011. Comparison of chondrogenic differentiation of adipose tissue-derived mesenchymal stem cells with cultured chondrocytes and bone marrow mesenchymal stem cells. Acta Chir. Orthop. Traumatol. Cech., 78: 138-144. PMID: 21575557
Hwang, Y., N. Sangaj and S. Varghese, 2010. Interconnected macroporous poly (ethylene glycol) cryogels as a cell scaffold for cartilage tissue engineering. Tissue Eng. Part A., 16: 3033-3041. DOI: 10.1089/ten.TEA.2010.0045

Kestendjieva, S., D. Kyurkchiev, G. Tsvetkova, T. Mehandjiev and A. Dimitrov et al., 2008. Characterization of mesenchymal stem cells isolated from the human umbilical cord. Cell Biol. Int., 32: 724-732. DOI: 10.1016/j.cellbi.2008.02.002

Ko, J.Y., K.I., Kim, S. Park and G.I. Im, 2014. In vitro chondrogenesis and in vivo repair of osteochondral defect with human induced pluripotent stem cells. Biomaterials, 35: 3571-81.

DOI: 10.1016/j.biomaterials.2014.01.009

Kuroda, R., K. Ishida, T. Matsumoto, T. Akisue and H. Fujioka et al., 2007. Treatment of a full-thickness articular cartilage defect in the femoral condyle of an athlete with autologous bone-marrow stromal cells. Osteoarthritis Cartilage, 15: 226-2231. DOI: 10.1016/j.joca.2006.08.008

Liu, M., X. Yu, F. Huang, S. Cen and G. Zhong et al., 2013. Tissue engineering stratified scaffolds for articular cartilage and subchondral bone defects repair. Orthopedics, 36: 868-873. DOI: $10.3928 / 01477447-20131021-10$

Malicev, E., N. Kregar-Velikonja, A. Barlic, A. Alibegović and M. Drobnic, 2009. Comparison of articular and auricular cartilage as a cell source for the autologous chondrocyte implantation. J. Orthop. Res., 27: 943-948. DOI: 10.1002/jor.20833

Mescher, A., 2013. Junqueira's Basic Histology: Text and Atlas. 13th Edn., McGraw Hill Professional, ISBN-10: 0071807209, pp: 480.

Mobasheri, A., C. Csaki, A.L. Clutterbuck, M. Rahmanzadeh and M. Shakibaei, 2009. Mesenchymal stem cells in connective tissue engineering and regenerative medicine: Applications in cartilage repair and osteoarthritis therapy. Histol. Histopathol., 24: 347-366. PMID: 19130405

Nejadnik, H., J.H. Hui, E.P. Feng Choong, B.C. Tai and E.H. Lee, 2010. Autologous bone marrow-derived mesenchymal stem cells versus autologous chondrocyte implantation: An observational cohort study. Am. J. Sports Med., 38: 1110-1116. DOI: $10.1177 / 0363546509359067$

Nooeaid, P., V. Salih, J.P. Beier and A.R. Boccaccini, 2012. Osteochondral tissue engineering: Scaffolds, stem cells and applications. J. Cell Mol. Med., 16: 2247-2270. DOI: 10.1111/j.1582-4934.2012.01571.x

Odabas, S., A.E. Elçin and Y.M. Elçin, 2014. Isolation and characterization of mesenchymal stem cells. Methods Mol. Biol., 1109: 47-63. DOI: 10.1007/978-1-4614-9437-9_3 
Shimizu, T., T. Ishikawa, E. Sugihara, S. Kuninaka and T. Miyamoto et al., 2010. C-MYC overexpression with loss of Ink4a/Arf transforms bone marrow stromal cells into osteosarcoma accompanied by loss of adipogenesis. Oncogene, 29: 5687-5699. DOI: $10.1038 /$ onc. 2010.312

Sophia Fox, A.J., A. Bedi and S.A. Rodeo, 2009. The basic science of articular cartilage: Structure, composition and function. Sports Health, 1: 461-468. PMID: 23015907

Takahashi, K. and S. Yamanaka, 2013. Induced pluripotent stem cells in medicine and biology. Development, 140: 2457-2461. DOI: $10.1242 /$ dev.092551

Takahashi, K., K. Tanabe, M. Ohnuki, M. Narita and T. Ichisaka et al., 2007. Induction of pluripotent stem cells from adult human fibroblasts by defined factors. Cell, 131: 861-872. DOI: 10.1016/j.cell.2007.11.019

Varga, I., D. Holly, J. Vojtassak, D. Bohmer and S. Polak et al., 2011. Morphological characterization of in vitro expanded human dental pulp-derived stem cells. Biologia, 66: 706-711. DOI: $10.2478 / \mathrm{s} 11756-011-0069-3$

Wilmut, I., A.E. Schnieke, J. McWhir, A.J. Kind and K.H. Campbell, 1997. Viable offspring derived from fetal and adult mammalian cells. Nature, 385: 810-813. DOI: $10.1038 / 385810 \mathrm{a} 0$

Xue, J.X., Y.Y. Gong, G.D. Zhou, W. Liu and Y. Cao et al., 2012. Chondrogenic differentiation of bone marrowderived mesenchymal stem cells induced by acellular cartilage sheets. Biomaterials, 33: 5832-5840.

DOI: 10.1016/j.biomaterials.2012.04.054
Yu, D.A., J. Han and B.S. Kim, 2012. Stimulation of chondrogenic differentiation of mesenchymal stem cells. Int. J. Stem. Cells, 5: 16-22. PMID: 24298351

Yu, J., M.A. Vodyanik, K. Smuga-Otto, J. AntosiewiczBourget and J.L. Frane et al., 2007. Induced pluripotent stem cell lines derived from human somatic cells. Science, 318: 1917-1920. PMID: 18029452

Zhang, L., J. Hu and K.A. Athanasiou, 2009a. The role of tissue engineering in articular cartilage repair and regeneration. Crit. Rev. Biomed. Eng., 37: 1-57. PMID: 20201770

Zhang, Y.N., P.C. Lie and X. Wei, 2009b. Differentiation of mesenchymal stromal cells derived from umbilical cord Wharton's jelly into hepatocyte-like cells. Cytotherapy, 11: 548-558. DOI: $10.1080 / 14653240903051533$

Zhang, L., T. Yuan, L. Guo and X. Zhang, 2012. An in vitro study of collagen hydrogel to induce the chondrogenic differentiation of mesenchymal stem cells. J. Biomed Mater Res. A, 100: 2717-2725. DOI: 10.1002/jbm.a.34194

Zheng, L., H.S. Fan, J. Sun, X.N. Chen and G. Wang et al., 2010. Chondrogenic differentiation of mesenchymal stem cells induced by collagen-based hydrogel: An in vivo study. J. Biomed Mater Res. A, 93: 783-792. DOI: $10.1002 / \mathrm{jbm} . \mathrm{a} .32588$ 